\title{
Hard-sphere and hard-disk freezing from the differential formulation of the generalized effective liquid approximation
}

\author{
C. F. Tejero and J. A. Cuesta \\ Facultad de Ciencias Físicas, Universidad Complutense de Madrid, E-28040 Madrid, Spain
}

(Received 31 August 1992)

\begin{abstract}
We apply the differential formulation of the generalized effective liquid approximation to the study of hard-sphere and hard-disk freezing. We show that the thermodynamic properties of the solid phase are rather insensitive to the compressibility factor of the fluid phase used to map the solid onto the effective liquid. The solid-fluid coexistence data instead are quite dependent on the equation of state describing the fluid phase. Very accurate results, as compared with the simulation data, are obtained for both the freezing of hard spheres and hard disks.
\end{abstract}

PACS number(s): 64.70.Dv, 64.10. $+\mathrm{h}$

\section{INTRODUCTION}

The freezing of hard spheres into perfect crystals is probably the most notable example of the successful application of the nonperturbative density functional theories (NPDFT) to the study of classical nonuniform systems. In all these theories the excess free energy of the solid is determined from the excess free energy of an effective liquid, the major differences between the various theories consisting in the form in which the solid is mapped onto the effective liquid. In the weighted density approximations (WDA [1,2], MWDA [3]) the density of the effective liquid is obtained by a nonlocal weighted average of the solid density. In the direct-correlationfunction (DCF) approaches (ELA [4], GELA [5]) the DCF of the solid is approximated by that of an effective liquid with a lower bulk density.

When applied to soft interactions the NPDFT fail in a greater or lesser extent in predicting the freezing into crystalline structures [6]. It has been argued [7] that the relatively large density change at coexistence of the hard-sphere freezing makes the NPDFT insensitive to physical details, which could explain why these various theories work particularly well for hard spheres but not for soft inverse-power potentials with only a small density change upon freezing. Another possible explanation [6] is that the range of the solid correlations is incorrectly described when the range of the interaction potential is increased. That is, the effective liquid which gives a good description of the amplitude of the solid correlations does not necessarily provide a good estimate of their range. The fact that the hard-sphere system is a particular case for which the free energy and the entropy cannot be distinguished has also been claimed as a possible explanation for the failure of the NPDFT when applied to softer interactions [8].

The lack of NPDFT for the freezing of soft spheres has been circumvented by shifting attention to hard-sphere perturbation schemes as approximations to the study of more realistic potentials $[8,9]$. The interest of the hardsphere NPDFT is thus twofold, providing a selfcontained approximation for hard potentials and a reference system for the study of continuous potentials.

In this paper we consider an alternative, differential, formulation of the generalized effective liquid approximation (GELA) of Lutsko and Baus [5] and apply it to the study of the hard-sphere and hard-disk freezing. As explained already elsewhere [5] this approximation gives very accurate free energies, fluid-solid coexistence data, and pressures for the hard-sphere solid when compared with the simulation results. It also leads to most of the remaining NPDFT when additional assumptions are introduced into the theory. In Sec. II we introduce the differential formulation of the GELA as applied to the fluid-solid transition. The thermodynamic and structural properties of the fluid phase and the one-parameter Gaussian approximation for the solid density needed for solving the GELA are introduced in Sec. III while the ideal free energy of the solid is briefly discussed in Sec. IV. The results for the hard-sphere and hard-disk freezing are presented in Sec. $\mathrm{V}$ and our conclusions are gathered in Sec. VI.

\section{THE DIFFERENTIAL FORMULATION OF THE GELA}

The generalized effective liquid approximation (GELA) proposed by Lutsko and Baus [5] is a NPDFT for the determination of the excess free energy of a system characterized by a nonuniform one-particle density (a solid, a liquid crystal, etc.) in terms of the excess free energy of some effective liquid. For a solid of density $\rho_{s}(\mathbf{r})$, the density of the effective liquid $\hat{\rho}$ which is used to represent the solid is a functional of $\rho_{s}(\mathbf{r})$, a dependence which will be indicated by square brackets as $\hat{\rho}\left[\rho_{s}\right]$. In the GELA this functional dependence is given by the following integral equation [5]:

$$
\lambda \widehat{\rho}\left[\lambda \rho_{s}\right]=\frac{\int d \mathbf{r} \rho_{s}(\mathbf{r}) \int d \mathbf{r}^{\prime} \rho_{s}\left(\mathbf{r}^{\prime}\right) \int_{0}^{\lambda} d \alpha \int_{0}^{\alpha} d \alpha^{\prime} C\left(\left|\mathbf{r}-\mathbf{r}^{\prime}\right| ; \hat{\rho}\left[\alpha^{\prime} \rho_{s}\right]\right)}{\rho_{s} V_{D} \int_{0}^{1} d \alpha \int_{0}^{\alpha} d \alpha^{\prime} \int d \mathbf{r} C\left(|\mathbf{r}| ; \alpha^{\prime} \hat{\rho}\left[\lambda \rho_{s}\right]\right)},
$$


where $C(|\mathbf{r}| ; \rho)$ is the (Ornstein-Zernike) DCF of the liquid phase of density $\rho$. In Eq. (2.1), $\rho_{s}$ is moreover the average number density of the solid phase $\rho_{s} V_{D}=\int d \mathbf{r} \rho_{s}(\mathbf{r})$, with $V_{D}$ the volume of the $D$ dimensional box, and $\lambda$ is a parameter $(0 \leq \lambda \leq 1)$ which connects a reference density [here, $\rho_{r}(\mathbf{r})=0$ ] to the solid density $\rho_{s}(\mathbf{r})$ through a linear path in the space of density functions: $\rho_{\lambda}(\mathbf{r})=\lambda \rho_{s}(\mathbf{r})$. The density of the effective liquid is determined by setting $\lambda=1$ in Eq. (2.1), which is a highly nonlinear integral equation for $\hat{\rho}$.

The solution of the GELA considered by Lutsko and Baus [5] is obtained by expanding $\hat{\rho}\left[\lambda \rho_{s}\right]$ around $\lambda=1$ as

$$
\hat{\rho}\left[\lambda \rho_{s}\right]=\lambda \sum_{n=0}^{\infty}(\lambda-1)^{n} a_{n}\left[\rho_{s}\right]
$$

with $\hat{\rho}\left[\rho_{s}\right]=a_{0}\left[\rho_{s}\right]$. Substituting this expansion into Eq. (2.1) and differentiating with respect to $\lambda$, a sequence of equations is obtained which, when $\lambda$ is set equal to 1 , allows determination of the expansion coefficients $a_{n}$. However, this determination is not straightforward since the first two equations of the sequence involve all the expansion coefficients $a_{n}$. The method proposed by Lutsko and Baus is to solve the GELA by successive approximations. First, by setting $a_{1}=a_{2}=\cdots=0$ and determining $a_{0}$ from the first equation of the sequence. Next, keeping only $a_{0}$ and $a_{1}$ in Eq. (2.2), which are then calculated from the first two equations of the sequence, and so on. The procedure is terminated when the change in $a_{0}$ [which is the only coefficient that matters in Eq. (2.2)] becomes small. Two drawbacks of this method are the convergence of Eq. (2.2) and the truncation needed to solve the first two equations of the sequence.

An alternative (differential) formulation of the GELA has recently been introduced in connection with the study of the isotropic-nematic transition of hard convex bodies [10]. In this formulation, the original integral equation (2.1) is transformed into a system of two ordinary differential equations using the following transformation steps. Differentiating twice Eq. (2.1) with respect to $\lambda$ we have

$$
\frac{\partial^{2}}{\partial \lambda^{2}}\left\{\lambda \psi\left(\hat{\rho}\left[\lambda \rho_{s}\right]\right)\right\}=\Phi\left(\hat{\rho}\left[\lambda \rho_{s}\right] ;\left[\rho_{s}\right]\right),
$$

where

$$
\begin{aligned}
\Phi\left(\hat{\rho}\left[\lambda \rho_{s}\right] ;\left[\rho_{s}\right]\right) \equiv & -\frac{1}{\rho_{s} V_{D}} \int d \mathbf{r} \rho_{s}(\mathbf{r}) \\
& \times \int d \mathbf{r}^{\prime} \rho_{s}\left(\mathbf{r}^{\prime}\right) C\left(\left|\mathbf{r}-\mathbf{r}^{\prime}\right| ; \hat{\rho}\left[\lambda \rho_{s}\right]\right)
\end{aligned}
$$

is related to the excess free energy per particle of the solid phase $\phi_{\mathrm{ex}}\left[\rho_{s}\right][5]$ as $\left(\beta=1 / k_{B} T\right)$

$$
\beta \phi_{\mathrm{ex}}\left[\rho_{s}\right]=-\int_{0}^{1} d \lambda \int_{0}^{\lambda} d \lambda^{\prime} \Phi\left(\hat{\rho}\left[\lambda^{\prime} \rho_{s}\right] ;\left[\rho_{s}\right]\right)
$$

and

$$
\psi(\hat{\rho}) \equiv \beta \phi_{\mathrm{ex}}(\hat{\rho})=\frac{1}{\hat{\rho}} \int_{0}^{\hat{\rho}} d \rho \int_{0}^{\rho} d \rho^{\prime} \int d \mathbf{r} C\left(|\mathbf{r}| ; \rho^{\prime}\right)
$$

with $\phi_{\text {ex }}(\hat{\rho})$ equal to the excess free energy per particle of the liquid phase of density $\hat{\rho}$. Equation (2.3) can be fur- ther transformed by taking into account that for fixed $\rho_{s}(\mathbf{r})$ all the functionals become ordinary functions of $\lambda$. Let $\hat{\eta}(\lambda)=v_{D} \hat{\rho}(\lambda)$ denote the packing fraction (the fraction of the total volume $V_{D}$ occupied by the $D$-spheres) with $v_{D}$ denoting the volume of a $D$-sphere of unit radius. With the definition

$$
z(\lambda) \equiv \frac{\partial}{\partial \lambda}\{\lambda \psi(\hat{\eta}(\lambda))\}
$$

Eq. (2.3) can be rewritten as

$$
z^{\prime}(\lambda)=\Phi(\hat{\eta}(\lambda))
$$

where the prime denotes the derivative with respect to $\lambda$. Moreover, computing the derivative indicated in the right-hand side of Eq. (2.7) and grouping terms we find

$$
\hat{\eta}^{\prime}(\lambda)=\frac{z(\lambda)-\psi(\hat{\eta}(\lambda))}{\lambda \psi^{\prime}(\hat{\eta}(\lambda))} .
$$

Notice that for $\lambda=0$ the apparent singular behavior of Eq. (2.9) can be regularized by considering the exact lowdensity expansion of $\psi(\hat{\eta}), \psi(\hat{\eta})=2^{D-1} \hat{\eta}+\cdots$, to obtain

$$
\hat{\eta}^{\prime}(0)=\frac{1}{2^{D}} z^{\prime}(0)=\frac{1}{2^{D}} \Phi(0) .
$$

The differential formulation of the GELA consists in the system of two ordinary differential equations in $\hat{\eta}(\lambda)$ and $z(\lambda)$ [Eqs. (2.8) and (2.9)] which together with the initial conditions [following from Eqs. (2.1) and (2.7)]

$$
\hat{\eta}(0)=z(0)=0
$$

can be solved numerically to obtain the effective liquid density $\hat{\eta}(1)$. The excess free energy of the solid phase $\phi_{\text {ex }}\left[\rho_{s}\right]$ can then finally be determined from

$$
\beta \phi_{\mathrm{ex}}\left[\rho_{s]}=\psi(\hat{\eta}(1)) .\right.
$$

\section{APPLICATION TO HARD SPHERES AND HARD DISKS}

In order to solve the ordinary differential equations of the GELA [Eqs. (2.8)-(2.11)] we need as input the thermodynamics, $\psi(\hat{\eta})$, and the structure, $C(|\mathbf{r}| ; \hat{\eta})$, of the liquid phase together with the periodic density of the solid, $\rho_{s}(\mathbf{r})$. For the latter we use the one-parameter Gaussian approximation

$$
\rho_{s}(\mathbf{r})=\left(\frac{\alpha}{\pi}\right)^{D / 2} \sum_{j} \exp \left[-\alpha\left(\mathbf{r}-\mathbf{r}_{j}\right)^{2}\right],
$$

where the sum runs over the Bravais lattice vectors $\left\{\mathbf{r}_{j}\right\}$ of the crystal structure.

Taking into account the translational invariance properties of the crystal lattice and performing the angular integrations in Eq. (2.4) we find

$\Phi(\widehat{\eta}(\lambda))=-\sum_{j} \int_{0}^{\infty} d R R C(R ; \widehat{\eta}(\lambda)) S\left(R ; \alpha, r_{j}\right)$

where 


$$
\begin{aligned}
S\left(R ; \alpha, r_{j}\right)=\left[\frac{\alpha}{2 \pi r_{j}^{2}}\right]^{1 / 2} & {\left[\exp \left[-\frac{\alpha}{2}\left(R-r_{j}\right)^{2}\right]\right.} \\
& \left.-\exp \left[-\frac{\alpha}{2}\left(R+r_{j}\right)^{2}\right]\right]
\end{aligned}
$$

for $D=3$ and

$$
S\left(R ; \alpha, r_{j}\right)=\alpha \exp \left[-\frac{\alpha}{2}\left(R^{2}+r_{j}^{2}\right)\right] I_{0}\left(\alpha R r_{j}\right)
$$

for $D=2$, with $I_{0}(x)$ denoting the zeroth-order modified Bessel function.

The thermodynamic and structural properties of the fluid phase can be obtained from different approximate theories. For the structure of the fluid phase, i.e., the DCF, we consider the Percus-Yevick (PY) equation which can be solved exactly for hard spheres [11] leading to

$C(x ; \hat{\eta})=-\Theta(1-x)\left[\gamma_{1}+6 \hat{\eta} \gamma_{2} x+\frac{1}{2} \hat{\eta} \gamma_{1} x^{3}\right]$,

where $\Theta(x)$ is the Heaviside step function, $\gamma_{1}$ $=(1+2 \hat{\eta})^{2} /(1-\hat{\eta})^{4}, \quad \gamma_{2}=-(1+\hat{\eta} / 2)^{2} /(1-\hat{\eta})^{4}, \quad \hat{\eta}=\pi$ $\sigma^{3} \hat{\rho} / 6$, and $x=R / \sigma$ with $\sigma$ the hard-sphere diameter. As the PY equation can only be solved analytically for the odd values of $D$, we take for the DCF of the harddisk fluid the approximate analytical expression of Baus and Colot [12] which compares very well to the numerical solution of the PY equation [13]. This expression is obtained by assuming that the low-density expansion of the DCF can be extended to the high-density regime if reformulated in terms of a rescaled density dependent diameter [12] leading to

$$
\begin{aligned}
C(x ; \hat{\eta})=-\frac{\partial}{\partial \hat{\eta}}\left[\hat{\eta} Z_{2}(\hat{\eta})\right] \Theta(1-x)[ & 1-a^{2} \hat{\eta} \\
& \left.+a^{2} \hat{\eta} \omega_{2}\left(\frac{x}{a}\right)\right],
\end{aligned}
$$

where $Z_{2}(\hat{\eta})=\left(1+c_{2} \hat{\eta}^{2}\right) /(1-\hat{\eta})^{2}, c_{2}=7 / 3-4 \sqrt{3} / \pi, \hat{\eta}$ $=\pi \sigma^{2} \hat{\rho} / 4$, and $x=R / \sigma$ with $\sigma$ the hard-disk diameter. Here $\omega_{2}(x / a)$ is related to the overlap "area" of two hard disks whose centers are a distance $x / a$ apart and is given by

$$
\omega_{2}\left(\frac{x}{a}\right)=\frac{2}{\pi}\left\{\arccos \left(\frac{x}{a}\right)-\frac{x}{a}\left[1-\left(\frac{x}{a}\right)^{2}\right]^{1 / 2}\right\},
$$

where $a=a(\hat{\eta})$ is the ratio of the effective to the real hard-disk diameter which is determined from the nonlinear equation

$$
\begin{aligned}
\frac{\pi}{2 \hat{\eta}^{2}} & {\left[1-4 \hat{\eta}-\left[\frac{\partial}{\partial \hat{\eta}}\left[\hat{\eta} Z_{2}(\hat{\eta})\right]\right]^{-1}\right] } \\
= & a^{2}\left(a^{2}-4\right) \arcsin \left[\frac{1}{a}\right]-\left(a^{2}+2\right)\left(a^{2}-1\right)^{1 / 2} .
\end{aligned}
$$

We have also investigated the effect of using different effective fluid equations of state on the thermodynamic properties of the solid phase. The excess energy per particle of the fluid phase is determined by

$$
\psi(\eta)=\int_{0}^{\eta} d \eta^{\prime} \frac{Z\left(\eta^{\prime}\right)-1}{\eta^{\prime}},
$$

where $Z(\eta)=\beta P / \rho$ is the compressibility factor and $P$ is the pressure. For hard spheres we have considered both the PY compressibility equation of state, $Z_{\mathrm{PY}}(\eta)$, and the Carnahan-Starling $(\mathrm{CS})$ equation, $Z_{\mathrm{CS}}(\eta)$. For hard disks we have looked for different compressibility factors by writing

$$
Z(\eta)=\frac{1+\sum_{n=1}^{\infty} c_{n} \eta^{n}}{(1-\eta)^{2}}
$$

an expression proposed by Baus and Colot [12] as a generalization of the results of scaled-particle theory. As explained therein the expansion coefficients $c_{n}$ are determined from the virial coefficients by expanding Eq. (3.10) into a virial series. In practice, the infinite series $\sum_{n=1}^{\infty} c_{n} \eta^{n}$ in Eq. (3.10) is truncated at some finite order $N$. We have considered two cases: $Z(\eta)=Z_{N}(\eta)$ with $N=2$ and $N=6$. Compared with the simulation data of Erpenbeck and Luban [14], it can be shown that $Z_{2}(\eta)$ is not accurate for large densities whereas $Z_{6}(\eta)$ is very close to the simulation results. Since it is needed only for low- $\hat{\eta}$ values, we have used $Z=Z_{2}(\eta)$ in Eq. (3.6) but as the fluid-solid coexistence data are very sensitive to the fluid equation of state, we have used both $Z_{2}(\eta)$ and $Z_{6}(\eta)$ for the equation of state of the fluid. Moreover, we have also considered an analytical compressibility factor in the same form as $Z_{6}(\eta)$ but with $c_{2}$ and $c_{3}$ determined from the virial coefficients $B_{3}$ and $B_{4}$ (which are known exactly) while $c_{4}, c_{5}$, and $c_{6}$ were fitted to the simulation data of Erpenbeck and Luban [14] yielding $c_{4}=-0.72553, c_{5}=2.52783$, and $c_{6}=-2.4945$. This compressibility factor will henceforth be denoted by $Z_{\mathrm{EL}}(\eta)$.

\section{THE IDEAL FREE ENERGY OF THE SOLID PHASE}

The ideal free energy per particle of the solid phase is given by

$$
\beta \phi_{\mathrm{id}}\left[\rho_{s}\right]=\frac{1}{\rho_{s} V_{D}} \int d \mathbf{r} \rho_{s}(\mathbf{r})\left\{\ln \left[\Lambda^{D} \rho_{s}(\mathbf{r})\right]-1\right\},
$$

where $\Lambda$ is the de Broglie thermal wavelength. More elaborate expressions for $\beta \phi_{i d}\left[\rho_{s}\right]$ using the one-parameter Gaussian approximation [Eq. (3.1)] can be found elsewhere for hard spheres [5] and hard disks [15] and will not be repeated here. We note instead that for the large$\alpha$ values where the equilibrium solids are found, the ideal free energy per particle of the solid phase can be approximated by its asymptotic large- $\alpha$ form,

$\beta \phi_{\text {id }}\left[\rho_{s}\right]=D \ln \left[\frac{\Lambda}{\sigma}\right]+\frac{D}{2}\left[\ln \left(\frac{\alpha \sigma^{2}}{\pi}\right)-1\right]-1$.

Once the total free energy particle of the solid phase 
$\beta f=\beta \phi_{\mathrm{id}}+\beta \phi_{\mathrm{ex}}$ is determined from Eqs. (2.12) and (4.2), the equilibrium solid is determined for a given average solid density by minimizing $\beta f$ with respect to the Gaussian width parameter $\alpha$ for each crystal structure. The resulting free energies are then used to determine the compressibility factor $Z$ (or pressure) and the chemical potential $\mu$ from the thermodynamic relations

$$
Z=\eta \frac{\partial(\beta f)}{\partial \eta}, \beta \mu=\frac{\partial}{\partial \eta}(\eta \beta f) \text {. }
$$

As indicated by Lutsko and Baus [5] we note that in finding the minimum of the total free energy versus $\alpha$ some structure develops in the small- $\alpha$ region which corresponds to large- $\hat{\eta}$ values. This structure appears only because the PY equation is not an accurate description for the structure of the fluid in the high-density region. It can be shown [5] that most of this small- $\alpha$ structure disappears when using an improved DCF for the fluid phase without changing the large- $\alpha$ behavior. As we are interested only in the large- $\alpha$ region, where the equilibrium solids are found, the PY DCF has been used in the present investigation. This will moreover allow us to test our results for hard spheres against the previous calculations which also did use the PY DCF for the study of the solid phase.

\section{RESULTS}

\section{A. Hard spheres}

Our results for the freezing of hard spheres are essentially the same as those obtained by Lutsko and Baus [5] using the $\lambda$-expansion method described in Sec. II. In Table I we compare the free energy per particle, $\beta \phi=\beta f-3 \ln (\Lambda / \sigma)+1$, and the pressure of the fcc and bcc hard-sphere solids (using $Z_{\mathrm{CS}}$ to map the solid onto the fluid) with some available simulation data $[2,16]$. It can be seen that the free energy is underestimated for the fcc solid while it is overestimated for the bcc solid, the relative errors being only $2 \%$. Moreover, the pressure is slightly overestimated for the compact lattice (about 2\%). We note that both the free energy and the pressure obtained from the differential formulation of the GELA are slightly higher than the results of Lutsko and Baus. We also remark that the solid free energy $\beta \phi$ is rather insensitive to the fluid compressibility factor (CS or PY) used to solve the GELA. For instance, the results for $\eta=0.50$ (0.60) are $\beta \phi=5.087$ (7.261) when $Z_{\mathrm{CS}}$ is used and 5.080

TABLE I. Free energy per particle $\beta \phi$ and compressibility factor $\beta P / \rho_{s}$ as computed from the differential formulation of the GELA for the fcc and bcc hard-sphere solids at various densities. The simulation results have been taken from Refs. $[2,16]$.

\begin{tabular}{ccccc}
\hline \hline$\rho \sigma^{3}$ & $\beta \phi(\mathrm{MC})$ & $\beta \phi$ & $\beta P / \rho_{s}(\mathrm{MC})$ & $\beta P / \rho_{s}$ \\
\hline $1.000(\mathrm{fcc})$ & 5.661 & 5.549 & 10.26 & 10.4 \\
1.025 (fcc) & 5.893 & 5.813 & 10.84 & 11.0 \\
1.050 (fcc) & 6.148 & 6.086 & 11.54 & 11.7 \\
$1.075(\mathrm{fcc})$ & 6.426 & 6.370 & 12.28 & 12.5 \\
1.100 (fcc) & 6.758 & 6.668 & 13.12 & 13.4 \\
1.041 (bcc) & 6.134 & 6.174 & & 14.7 \\
1.100 (bcc) & 6.973 & 7.105 & & 19.6 \\
\hline \hline
\end{tabular}

(7.257) when $Z_{\mathrm{PY}}$ is used.

By considering the three phases two by two the exchange of stability is found to occur at $\eta=0.517$ (fluidfcc), at $\eta=0.528$ (fluid-bcc), and at $\eta=0.503$ (fcc-bcc). These results show that the bcc solid is metastable in the whole density range: with respect to the fluid phase in the low-density region and with respect to the fcc solid in the high-density region.

Finally, the fluid-fcc solid coexistence data obtained from the differential formulation of the GELA are $\eta_{F}=0.496(0.471), \eta_{s}=0.545 \quad(0.522)$, and $P^{*}=\beta P \sigma^{3}$ $=12.0(10.3)$ when the CS (PY) equation of state is used for the fluid phase. In the former case our results give very accurate results (the reduced pressure $P^{*}$ being slightly overestimated) as compared with the simulation data [17] $\left(\eta_{F}=0.494, \eta_{S}=0.545\right.$, and $\left.P^{*}=11.7\right)$, the major difference with respect to the results of Lutsko and Baus [5] being the improvement of the value of the Lindemann ratio at coexistence: $L=0.112$ in this work and $L=0.100$ in [5]. In view of the relative insensitivity of the solid free energy with respect to the fluid used to map the solid onto the effective liquid, it becomes apparent that the quality of the fluid-solid coexistence data are mainly determined by the quality of the equation of state of the fluid phase.

\section{B. Hard disks}

The melting of two-dimensional solids is a controversial question. Following Kosterlitz, Thouless, Halperin, Nelson, and Young [18] the melting might proceed via two continuous transitions involving an intermediate phase (the hexatic phase) connecting the solid and the fluid phases. A transition of the usual first-order type, as observed in three-dimensional systems, however, has been proposed by several authors. In spite of a large body of simulation and experimental research, the nature of the two-dimensional freezing is still unknown.

In the present investigation the solid-fluid transition is assumed to proceed via a first-order transition as for $D=3$. As usual, the free energies of the fluid and solid phases are determined separately and the two-phase coexistence data are obtained from the solution of the equilibrium coexistence conditions $\mu_{F}\left(\eta_{F}\right)=\mu_{S}\left(\eta_{S}\right)$ and $P_{F}\left(\eta_{F}\right)=P_{S}\left(\eta_{S}\right)$

We have considered both the square lattice and the triangular lattice. In the former case no mechanically stable solid has been found (there is no free-energy minimum with respect to the Gaussian parameter $\alpha$ ). For the compact structure stable solids are found for packing fractions $\eta \geq 0.700$. In Fig. 1 the free energy per particle of the solid phase, $\beta \phi=\beta f-2 \ln (\Lambda / \sigma)+1$, is represented versus $Y \equiv\left(\alpha \sigma^{2}\right)^{1 / 2}$ for different packing fractions. It is found that $\beta \phi$ is rather insensitive with respect to the compressibility factor used to solve the GELA. Indeed, for $\eta=0.70(0.74)$ we have $\beta \phi=3.525$ (4.101), 3.519 (4.097), and 3.521 (4.098), for $Z=Z_{2}, Z_{6}$, and $Z_{\mathrm{EL}}$, respectively.

In Table II the solid-fluid coexistence data obtained from the different equations of state of the fluid phase are compared with the MD simulation data of Alder and oth- 


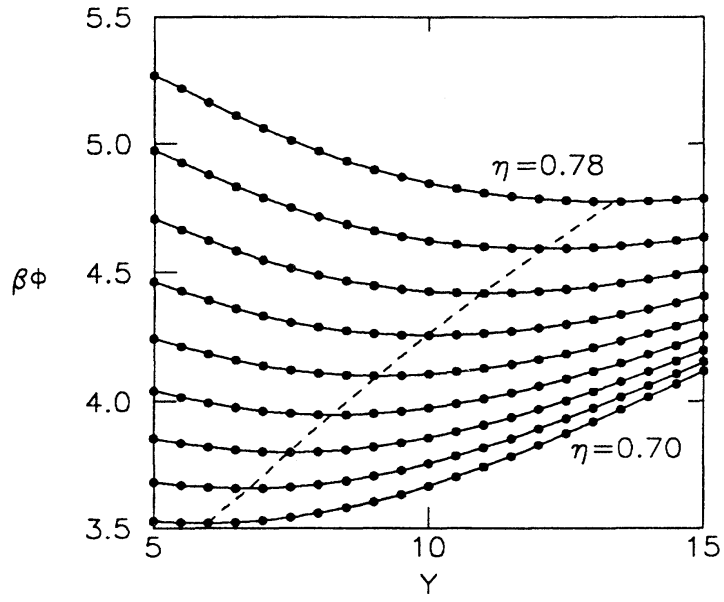

FIG. 1. Free energy per particle $\beta \phi$ vs $Y=\left(\alpha \sigma^{2}\right)^{1 / 2}$ of a compact hard-disk solid of packing fraction $\eta=0.70-0.78$, by steps of 0.01 (from bottom to top). The dashed line indicates the location of the minimum corresponding to the stable solid.

ers [19] and the MC simulation results of Hoover and Ree [17]. We have also included theoretical results obtained from the ELA [20] and the MWDA [21]. It is seen that the results obtained using $Z_{\mathrm{EL}}$ to describe the fluid phase compare favorably with the simulation results, while there is less good agreement when using $Z_{2}$ or $Z_{6}$. Notice that the theoretical results of the ELA [20]

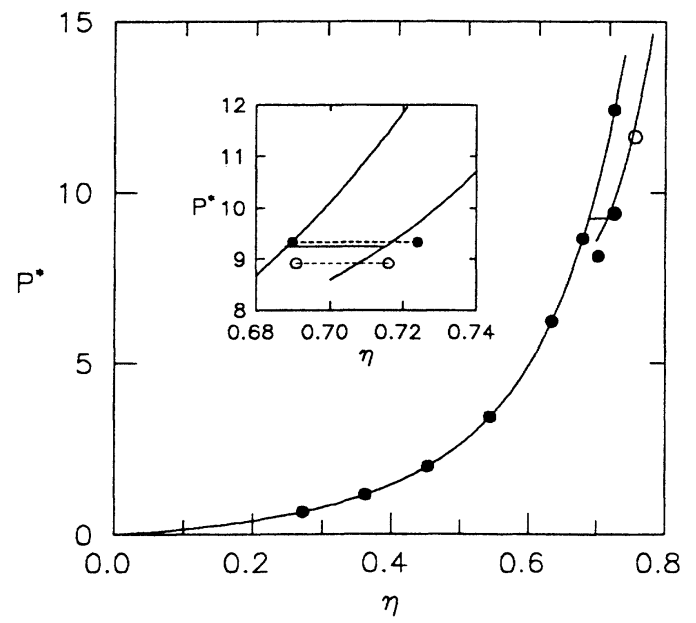

FIG. 2. The solid-fluid phase diagram of hard disks as obtained from the differential formulation of the GELA in the $P^{*}-\eta$ plane, with $P^{*}=\beta P \sigma^{2}$. The fluid branch has been obtained by fitting the MC data of Erpenbeck and Luban [14] with $Z_{\mathrm{EL}}$. The solid branch is the result of the present investigation. The horizontal line connecting the solid and fluid branches corresponds to the coexistence data (obtained with $Z_{\mathrm{EL}}$ ) appearing in Table II. The MC simulation results of Hoover and Ree [17] and the MD data of Alder and others [19] are denoted by solid and open dots, respectively. The inset shows the coexistence region in more detail. The three tie lines shown correspond to the results of [17] and [19] for the dashed lines and to the results of the GELA $\left(Z_{E L}\right)$ for the solid line.
TABLE II. Solid-fluid coexistence data as computed from different NPDFT $[20,21]$ of hard-disk freezing and compared to the simulation results $[17,19]$. Here $\eta_{F}$ and $\eta_{S}$ denote the coexisting packing fractions of the fluid and solid phases while $\beta P / \rho_{\mathrm{CP}}$, with $\rho_{\mathrm{CP}}$ the close-packing density, is the dimensionless pressure at coexistence.

\begin{tabular}{lccc}
\hline \hline & $\eta_{F}$ & $\eta_{S}$ & $\beta P / \rho_{\mathrm{CP}}$ \\
\hline MD [19] & 0.691 & 0.716 & 7.72 \\
MC [17] & 0.690 & 0.724 & 8.08 \\
GELA $\left(Z_{\mathrm{EL}}\right)$ & 0.688 & 0.715 & 8.0 \\
GELA $\left(Z_{6}\right)$ & 0.684 & 0.710 & 7.8 \\
GELA $\left(Z_{2}\right)$ & 0.674 & 0.700 & 7.4 \\
ELA [20] & 0.713 & 0.730 & 10.2 \\
MWDA [21] & 0.674 & 0.724 & 7.0 \\
\hline \hline
\end{tabular}

have been obtained with $Z_{2}$ while those of the MWDA [21] with $Z=(1-\eta)^{-2}$. The Lindemann ratio at coexistence found in these NPDFT is 0.177 (ELA) [20], 0.129 (MWDA) [21], and 0.119 in the present work.

In Fig. 2 the hard-disk phase diagram as obtained from the GELA using $Z_{\mathrm{EL}}$ for the compressibility factor of the fluid phase is compared with some available simulation results $[17,19]$. It is seen that the pressure of the solid phase obtained from our theoretical calculations is overestimated with respect to the simulation results, the relative error (about 3-7\%) decreasing as density increases.

\section{CONCLUSIONS}

The hard-sphere and hard-disk freezing has been obtained from the numerical solution of the differential formulation of the GELA. We have used a one-parameter Gaussian approximation for the periodic density of the solid phase and PY-like DCF for the effective liquid. Different equations of state for the fluid phase have been used to determine the solid-fluid coexistence data. We have shown that the free energy of the solid phase is rather insensitive to the compressibility factor used to map the solid onto the liquid. However, the packing fraction of the coexisting solid and fluid phases and the pressure at coexistence depend strongly on the equation of state of the fluid phase. When the CS equation for $D=3$ and the compressibility factor $Z_{\mathrm{EL}}$ obtained from the MC simulation data of Erpenbeck and Luban for $D=2$ are used we obtain very accurate results as compared with the available simulation coexistence data.

In conclusion, we have shown that the predictions of the GELA are in good agreement with the computer simulations whenever the fluid is described by an "exact" equation of state. On the other hand, the thermodynamic properties of the solid are rather insensitive to the fluid data used as input to map the solid onto the liquid.

\section{ACKNOWLEDGMENTS}

We are grateful to M. Baus for useful discussions. This work has been supported by the Dirección General de Investigación Cientǐfica y Tećnica (DGICYT, Spain) (PB91-0378). 
[1] P. Tarazona, Mol. Phys. 52, 81 (1984); Phys. Rev. A 31, 2672 (1985).

[2] W. A. Curtin and N. W. Ashcroft, Phys. Rev. A 32, 2909 (1985).

[3] A. R. Denton and N. W. Ashcroft, Phys. Rev. A 39, 4701 (1989).

[4] M. Baus and J. L. Colot, Mol. Phys. 55, 653 (1985).

[5] J. F. Lutsko and M. Baus, Phys. Rev. Lett. 64, 761 (1990); Phys. Rev. A 41, 6647 (1990).

[6] B. B. Laird and D. M. Kroll, Phys. Rev. A 42, 4810 (1990).

[7] Y. Rosenfeld, Phys. Rev. A 43, 5424 (1991).

[8] J. F. Lutsko and M. Baus, J. Phys. Condens. Matter 3, 6547 (1991).

[9] C. F. Tejero, J. F. Lutsko, J. L. Colot, and M. Baus, Phys. Rev. A 46, 3373 (1992).

[10] J. A. Cuesta, C. F. Tejero, and M. Baus, Phys. Rev. A 45, 7395 (1992).

[11] J. P. Hansen and I. R. McDonald, Theory of Simple
Liquids (Academic, London, 1986).

[12] M. Baus and J. L. Colot, Phys. Rev. A 36, 3912 (1987).

[13] F. Lado, J. Chem. Phys. 49, 3092 (1968).

[14] J. J. Erpenbeck and M. Luban, Phys. Rev. A 32, 2920 (1985).

[15] H. Xu, Ph.D. thesis, Université Libre de Bruxelles (unpublished).

[16] W. A. Curtin and K. Runge, Phys. Rev. A 35, 4755 (1987).

[17] W. G. Hoover and F. H. Ree, J. Chem. Phys. 49, 3609 (1968).

[18] K. J. Strandburg, Rev. Mod. Phys. 60, 161 (1988).

[19] B. J. Adler and T. E. Wainwright, Phys. Rev. 127, 359 (1962); B. J. Alder, W. G. Hoover, and D. A. Young, J. Chem. Phys. 49, 3688 (1968).

[20] J. L. Colot and M. Baus, Phys. Lett. A 119, 135 (1986).

[21] X. C. Zeng and D. W. Oxtoby, J. Chem. Phys. 93, 2692 (1990). 